\title{
A BOUNDARY INTEGRAL EQUATION METHOD FOR THE NUMERICAL SOLUTION OF A SECOND ORDER ELLIPTIC EQUATION WITH VARIABLE COEFFICIENTS
}

\author{
D. L. CLEMENTS
}

(Received 15 October 1979)

(Revised 14 December 1979)

\begin{abstract}
A method is derived for the solution of boundary value problems governed by a second-order elliptic partial differential equation with variable coefficients. The method is obtained by expressing the solution to a particular problem in terms of an integral taken round the boundary of the region under consideration.
\end{abstract}

\section{Introduction}

The boundary integral equation method has been shown to be an effective technique for the numerical solution of boundary value problems governed by linear elliptic partial differential equations with constant coefficients (see, for example, Cruse and Lachat [4], Cruse and Rizzo [5], Zienkiewicz et al. [6]). The method consists of expressing the solution to a particular boundary value problem in terms of an integral taken round the boundary of the region under consideration. The numerical solution of this integral equation is then obtained by replacing the integral by a sum and solving the resulting system of linear algebraic equations. Hence, since the method involves the discretization of only the boundary and not the whole domain, it offers some advantages over the finite element and finite difference procedures.

The aim of the present paper is to derive a boundary integral equation method for the solution of a second-order linear elliptic partial differential equation with variable coefficients. The equation considered governs certain important classes of thermostatic, elastostatic and electrostatic problems and hence the method provides an alternative to existing numerical procedures for a significant class of problems. A particular numerical example is considered in order to demonstrate the application of the method. 


\section{The boundary value problem}

Consider the elliptic partial differential equation

$$
\frac{\partial}{\partial x}\left[K(x) \frac{\partial \phi}{\partial x}\right]+\frac{\partial}{\partial y}\left[K(x) \frac{\partial \phi}{\partial y}\right]=0
$$

where $K(x)$ is a known function of the independent variable $x$. A solution to (2.1) is required which is valid in a region $R$ in $E^{2}$ with boundary $C$ which consists of a finite number of piecewise smooth closed curves. On $C$, either the dependent variable $\phi$ is specified or $\partial \phi / \partial n$ is specified, where $\mathbf{n}$ denotes the outward pointing normal to $C$.

Problems governed by equation (2.1) typically occur in plane inhomogeneous thermostatics or elastostatics with the independent variables $x$ and $y$ denoting the coordinates of a point in a Cartesian frame and $\phi$ being the temperature or displacement. In such applications the function $K(x)$ would represent a material parameter. From a practical viewpoint it is therefore reasonable to require that $K(x)$ be nonzero throughout the region $R$ under consideration. Further, $K(x)$ will be required to be continuous and have continuous derivatives in $R$.

Although in the current discussion attention will be restricted to the equation (2.1) it is appropriate to note that the more general equation

$$
\frac{\partial^{2} U}{\partial \xi^{2}}+a(\xi) \frac{\partial U}{\partial \xi}+b(\xi) U+c(\xi) \frac{\partial^{2} U}{\partial \eta^{2}}=0
$$

with $c(\xi)>0$, can be transformed readily into (2.1) by a simple change of the independent and dependent variables (see Clements and Rogers [3] for details). Hence a number of boundary value problems for equations of the type (2.2) may be solved readily by employing the procedure outlined in this paper.

The method of solution will be to express the solution to the boundary value problem in terms of an integral taken round the boundary $C$ of the region $R$ under consideration. Standard numerical procedures may then be used to solve the resulting integral equation.

\section{Solution of (2.1) in terms of an arbitrary harmonic function}

In (2.1) set

to obtain

$$
\phi(x, y)=[K(x)]^{-\frac{1}{2}} \psi(x, y)
$$

where

$$
\nabla^{2} \psi-\Lambda(x) \psi=0
$$

$$
\Lambda(x)=\frac{d}{d x}\left[\left(\frac{d K}{d x}\right)^{2} / 4 K\right] /\left(\frac{d K}{d x}\right)
$$


and

$$
\nabla^{2} \equiv \frac{\partial^{2}}{\partial x^{2}}+\frac{\partial^{2}}{\partial y^{2}}
$$

Consider the possibility of finding a solution to (3.2) in the form

$$
\psi=\sum_{n=0}^{\infty} h_{n}(x) F_{n}(x, y)
$$

where the $F_{n}$ are harmonic functions. Substitution of (3.4) into the left-hand side of (3.2) yields

$$
\nabla^{2} \psi-\Lambda(x) \psi=\sum_{n=0}^{\infty}\left[\left(\frac{d^{2} h_{n}}{d x^{2}}-\Lambda h_{n}\right) F_{n}+2 \frac{d h_{n}}{d x} \frac{\partial F_{n}}{\partial x}\right]
$$

Hence (3.4) will be a solution to (3.2) if the $h_{n}(x)$ and $F_{n}(x, y)$ satisfy the equations

$$
\frac{\partial F_{n}}{\partial x}=F_{n-1} \text { for } n \geqslant 1
$$

and

$$
2 \frac{d h_{n+1}}{d x}+\frac{d^{2} h_{n}}{d x^{2}}-\Lambda h_{n}=0 \quad \text { for } n \geqslant 0 \quad\left(h_{0} \text { constant }\right) .
$$

The functions $F_{n}$ in (3.6) are harmonic functions of $x$ and $y$. For the present purposes it is convenient to represent these harmonic functions as the real parts of analytic functions of the complex variable $z=x+i y$. Specifically, consider the functions $\Phi_{n}(z)$ such that $\Phi_{0}(z)$ is an arbitrary analytic function with

$$
\Phi_{n}(z)=\int_{0}^{z} \Phi_{n-1}(t) d t \quad \text { for } n=1,2, \ldots,
$$

and

$$
F_{n}(x, y)=\mathscr{R}\left\{\Phi_{n}(z)\right\} \quad \text { for } n=1,2, \ldots,
$$

where $\mathscr{R}$ denotes the real part of a complex number. Then, using (3.8),

$$
\frac{\partial F_{n}}{\partial x}=\mathscr{R}\left\{\frac{d \Phi_{n}}{d z}\right\}=\mathscr{R}\left\{\Phi_{n-1}\right\}=F_{n-1},
$$

showing that the $F_{n}$ given by (3.9) satisfy (3.6). Now, from (3.8),

$$
\Phi_{n}(z)=\frac{1}{(n-1) !} \int_{0}^{z}(z-t)^{n-1} \Phi_{0}(t) d t \text { for } n \geqslant 1,
$$

so that

$$
F_{n}(x, y)=\frac{1}{(n-1) !} \mathscr{R}\left\{\int_{0}^{z}(z-t)^{n-1} \Phi_{0}(t) d t\right\}
$$


Hence the solution to (3.2) takes the form

$$
\psi(x, y)=\mathscr{R}\left\{h_{0} \Phi_{0}(z)+\sum_{n=1}^{\infty} \frac{h_{n}(x)}{(n-1) !} \int_{0}^{z}(z-t)^{n-1} \Phi_{0}(t) d t\right\}
$$

Thus, from (3.1) and (3.12),

$$
\phi(x, y)=[K(x)]^{-\frac{1}{2}} \mathscr{R}\left\{h_{0} \Phi_{0}(z)+\sum_{n=1}^{\infty} \frac{h_{n}(x)}{(n-1) !} \int_{0}^{z}(z-t)^{n-1} \Phi_{0}(t) d t\right\} .
$$

Equation (3.13) provides the required solution to (2.1) in any domain in which the infinite series converges uniformly. The uniform convergence of the series may be investigated after the manner of Bergman [1]. Here it will suffice to note that for certain nontrivial cases the series in (3.13) truncates after a finite number of terms and in such cases (3.13) certainly provides the required solution to (2.1). For example, if the series terminates after only one term, so that $h_{n}=0$ for $n>0$, then (3.7) yields $\Lambda=0$ and (3.3) provides

$$
K(x)=(\alpha x+\beta)^{2},
$$

where $\alpha$ and $\beta$ are constants. Hence, if $K(x)$ is given by (3.14), then equation (2.1) admits a solution in terms of an arbitrary analytic function $\phi_{0}(z)$ in the form

$$
\phi(x, y)=(\alpha x+\beta)^{-1} \mathscr{R}\left\{h_{0} \Phi_{0}(z)\right\},
$$

where $h_{0}$ is an arbitrary constant. In the case when the series terminates after two terms, so that $h_{n}=0$ for $n>1$, equation (3.7) yields

$$
\Lambda=\frac{2 d h_{1} / d x}{h_{0}}
$$

and

$$
\begin{aligned}
\frac{d^{2} h_{1}}{d x^{2}} & =\Lambda h_{1} \\
& =\frac{2 h_{1} d h_{1} / d x}{h_{0}} .
\end{aligned}
$$

Hence

$$
h_{1}=\frac{-\gamma h_{0}}{\gamma x+\delta}
$$

and

$$
\Lambda=\frac{2 \gamma^{2}}{(\gamma x+\delta)^{2}},
$$

where $\gamma$ and $\delta$ are constants. From (3.3) and (3.19) it.now follows that

$$
K(x)=(\gamma x+\delta)^{-2}
$$


Thus, if $K(x)$ is given by (3.20), then equation (2.1) admits a solution in terms of an arbitrary analytic function $\Phi_{0}(z)$ in the form

$$
\phi(x, y)=(\gamma x+\delta) \mathscr{R}\left\{h_{0} \Phi_{0}(z)-\frac{\gamma h_{0}}{\gamma x+\delta} \int_{0}^{z} \Phi_{0}(t) d t\right\} .
$$

More generally, if $K(x)$ has the form

$$
K(x)=(\alpha x+\beta)^{p},
$$

then

$$
\Lambda(x)=\frac{p \alpha^{2}}{4}(p-2)(\alpha x+\beta)^{-2}
$$

so that, taking $h_{0}=1$,

$$
\begin{aligned}
& h_{1}(x)=\frac{-\alpha}{8} p(p-2)(\alpha x+\beta)^{-1} \\
& h_{2}(x)=\frac{\alpha^{2}}{128} p(p-2)(p+2)(p-4)(\alpha x+\beta)^{-2} \\
& \vdots \\
& h_{n}(x)=\frac{(-1)^{n} \alpha^{n}}{2^{3 n} n} p(p-2 n) \prod_{r=1}^{n-1}\left[p^{2}-(2 r)^{2}\right](\alpha x+\beta)^{-n} .
\end{aligned}
$$

Hence, from (3.13),

$$
\begin{aligned}
\phi(x, y)=(\alpha x+\beta)^{-\frac{1}{1} p} \mathscr{R}\left\{h_{0} \Phi_{0}(z)\right\} \\
+\sum_{n=1}^{\infty}\left\{\frac{(-1)^{n} \alpha^{n}}{2^{3 n} n} p(p-2 n) \prod_{r=1}^{n-1}\left[p^{2}-(2 r)^{2}\right]\right. \\
\left.\quad \times(\alpha x+\beta)^{-n} \int_{0}^{z}(z-t)^{n-1} \Phi_{0}(t) d t\right\} .
\end{aligned}
$$

It is clear that, if $p=2 N$ for $N=0, \pm 1, \pm 2$, then the series for $\phi$ truncates after a finite number of terms.

\section{A reciprocal theorem}

THEOREM. Let $\phi$ be a solution of

$$
\frac{\partial}{\partial x}\left[K(x) \frac{\partial \phi}{\partial x}\right]+\frac{\partial}{\partial y}\left[K(x) \frac{\partial \phi}{\partial y}\right]=0
$$

valid in the region $R$ in $E^{2}$ bounded by the contour $C$ consisting of a finite number of 
piecewise smooth closed curves. Also, let $\phi^{\prime}$ be another solution of (4.1) valid in $R$. Then

$$
\int_{C} K(x)\left[\frac{\partial \phi}{\partial n} \phi^{\prime}-\frac{\partial \phi^{\prime}}{\partial n} \phi\right] d s=0 .
$$

ProOF.

$$
\begin{aligned}
\int_{C} K(x) \frac{\partial \phi}{\partial n} \phi^{\prime} d s= & \int_{C} K(x)\left[\frac{\partial \phi}{\partial x} n_{1}+\frac{\partial \phi}{\partial y} n_{2}\right] \phi^{\prime} d s \\
= & \int_{R}\left\{\frac{\partial}{\partial x}\left[K(x) \frac{\partial \phi}{\partial x} \phi^{\prime}\right]+\frac{\partial}{\partial y}\left[K(x) \frac{\partial \phi}{\partial y} \phi^{\prime}\right]\right\} d R \\
= & \int_{R}\left[\left\{\frac{\partial}{\partial x}\left[K(x) \frac{\partial \phi}{\partial x}\right]+\frac{\partial}{\partial y}\left[K(x) \frac{\partial \phi}{\partial y}\right]\right\} \phi^{\prime}\right. \\
& \left.+K(x)\left\{\frac{\partial \phi}{\partial x} \frac{\partial \phi^{\prime}}{\partial x}+\frac{\partial \phi}{\partial y} \frac{\partial \phi^{\prime}}{\partial y}\right\}\right] d R
\end{aligned}
$$

Similarly,

$$
\begin{gathered}
\int_{C} K(x) \frac{\partial \phi^{\prime}}{\partial n} \phi d s=\int_{R}\left[\left\{\frac{\partial}{\partial x}\left[K(x) \frac{\partial \phi^{\prime}}{\partial x}\right]+\frac{\partial}{\partial y}\left[K(x) \frac{\partial \phi^{\prime}}{\partial y}\right]\right\} \phi\right. \\
\left.+K(x)\left\{\frac{\partial \phi}{\partial x} \frac{\partial \phi^{\prime}}{\partial x}+\frac{\partial \phi}{\partial y} \frac{\partial \phi^{\prime}}{\partial y}\right\}\right] d R .
\end{gathered}
$$

The required result is immediately obtained by subtracting (4.4) from (4.3) and using (4.1).

\section{The integral equation}

In (4.1) let $\phi$ denote a required solution to a boundary value problem governed by (2.1), and let $\phi^{\prime}$ be the solution given by (3.13) with

$$
\Phi_{0}(z)=\frac{1}{2 \pi} \log \left(z-z_{0}\right),
$$

where $z_{0}=a+i b$ is a point in $R$. Hence

$$
\phi^{\prime}=\frac{1}{2 \pi}[K(x)]^{-\frac{1}{2}} \mathscr{R}\left\{h_{0} \log \left(z-z_{0}\right)+\sum_{n=1}^{\infty} \frac{h_{n}(x)}{(n-1) !} \int_{0}^{z}(z-t)^{n-1} \log \left(t-z_{0}\right) d t\right\} .
$$

If (4.2) is to be valid with $\phi^{\prime}$ given by (5.2), then it is necessary to exclude the point $(a, b)$ by surrounding it with a small circle $\Gamma$ of radius $\varepsilon$. Then (4.2) yields

$$
\int_{C+\Gamma} K(x)\left[\frac{\partial \phi}{\partial n} \phi^{\prime}-\frac{\partial \phi^{\prime}}{\partial n} \phi\right] d s=0 .
$$


Now, on $\Gamma$,

$$
x=a+\varepsilon \cos \theta, \quad y=b+\varepsilon \sin \theta, \quad z=z_{0}+\varepsilon \exp (i \theta),
$$

so that

$$
\begin{aligned}
& \phi^{\prime}=\frac{1}{2 \pi[K(a+\varepsilon \cos \theta)]^{\frac{z}{2}}}\left\{h_{0} \log \varepsilon+\mathscr{R} \sum_{n=1}^{\infty} \frac{h_{n}(a+\varepsilon \cos \theta)}{(n-1) !}\right. \\
& \left.\times \int_{0}^{z_{0}+\varepsilon \exp (i \theta)}\left[z_{0}+\varepsilon \exp (i \theta)-t\right]^{n-1} \log \left(t-z_{0}\right) d t\right\} .
\end{aligned}
$$

It may be verified readily that, on $\Gamma$, for small $\varepsilon$,

$$
\begin{aligned}
\phi^{\prime} & =\frac{1}{2 \pi} \frac{h_{0}}{[K(a)]^{\frac{1}{2}}} \log \varepsilon+O(\varepsilon \log \varepsilon) \\
\frac{\partial \phi^{\prime}}{\partial n} & =\frac{-1}{2 \pi} \frac{h_{0}}{[K(a)]^{\frac{1}{2}}} \frac{1}{\varepsilon}+O(\log \varepsilon) .
\end{aligned}
$$

Hence, for small $\varepsilon$,

$$
\begin{aligned}
\int_{\Gamma} K(x) \frac{\partial \phi^{\prime}}{\partial n} \phi d s= & \frac{\dot{-1}}{2 \pi} \frac{h_{0}}{[K(a)]^{\frac{1}{2}}} \\
& \quad \times \int_{0}^{2 \pi} K(a+\varepsilon \cos \theta) \phi(a+\varepsilon \cos \theta, b+\varepsilon \sin \theta) d \theta+O(\varepsilon \log \varepsilon) \\
= & -h_{0}[K(a)]^{\frac{1}{2}} \phi(a, b)+O(\varepsilon \log \varepsilon) .
\end{aligned}
$$

Also, if $\partial \phi / \partial n$ is required to be bounded in $R$, then, since $K(x)$ is also bounded in $R$, it immediately follows from (5.5) that, for small $\varepsilon$,

$$
\int_{\Gamma} K(x) \frac{\partial \phi}{\partial n} \phi^{\prime} d s=O(\varepsilon \log \varepsilon)
$$

Hence

$$
\lim _{\varepsilon \rightarrow 0} \int_{\Gamma} K(x)\left[\frac{\partial \phi}{\partial n} \phi^{\prime}-\frac{\partial \phi^{\prime}}{\partial n} \phi\right] d s=h_{0}[K(a)]^{\frac{1}{2}} \phi(a, b) .
$$

Thus (5.3) yields

$$
\phi(a, b)=\frac{-1}{h_{0}[K(a)]^{\frac{1}{2}}} \int_{C} K(x)\left[\frac{\partial \phi}{\partial n} \phi^{\prime}-\frac{\partial \phi^{\prime}}{\partial n} \phi\right] d s .
$$

Equation (5.10) provides the required integral equation which expresses the solution to a particular boundary value problem in terms of an integral taken round the boundary of the region under consideration. If the point $(a, b)$ is on the boundary 
$C$ of the region $R$, then (5.10) should be replaced by

$$
\lambda \phi(a, b)=\frac{-1}{h_{0}[K(a)]^{\frac{1}{2}}} \int_{C} K(x)\left[\frac{\partial \phi}{\partial n} \phi^{\prime}-\frac{\partial \phi^{\prime}}{\partial n} \phi\right] d s,
$$

where $\lambda$ is a constant with $0<\lambda<1$. If $C$ has a continuously turning tangent then $\lambda=\frac{1}{2}$.

In general, (5.10) will not readily provide a useful analytical solution to a wellposed boundary value problem. However, it may be used to obtain a numerical solution to a particular boundary-value problem as follows. Suppose $\phi$ is given on $C$. Then (5.11) may be considered to be an integral equation for the unknown $\partial \phi / \partial n$ on $C$. The integral is replaced by a sum and $\partial \phi / \partial n$ determined at a number of points of the boundary. Equation (5.10) then yields a numerical value of $\phi$ at any interior point of interest. Alternatively, if $\partial \phi / \partial n$ is given on $C$, then (5.11) may be treated as an integral equation to determine $\phi$ on $C$. Once this has been done equation (5.10) yields $\phi$ at any interior point.

\section{A numerical example}

To demonstrate the numerical procedure, consider the following boundary value problem. Find a solution to

$$
\frac{\partial}{\partial x}\left[x^{-2} \frac{\partial \phi}{\partial x}\right]+\frac{\partial}{\partial y}\left[x^{-2} \frac{\partial \phi}{\partial y}\right]=0
$$

which is valid in the square $1<x<2,1<y<2$, and is subject to the boundary conditions

$$
\begin{array}{ll}
\phi=y, & \text { on } x=1, \\
\phi=y, & \text { on } x=2, \\
\phi=1, & \text { on } y=1, \\
\phi=2, & \text { on } y=2 .
\end{array}
$$

This problem admits the analytical solution $\phi=y$ which may be obtained from (3.21) by putting $\delta=0, \beta=h_{0}=1$ and $\Phi_{0}=i$.

For the particular equation (6.1), $K(x)=x^{-2}$, so that the integral equation (5.10) for the numerical solution of the problem is

$$
\lambda \phi(a, b)=-a \int_{C} x^{-2}\left[\frac{\partial \phi}{\partial n} \phi^{\prime}-\frac{\partial \phi^{\prime}}{\partial n} \phi\right] d s,
$$

where $\lambda=1$ if $(a, b) \in R$ and $0<\lambda<1$ if $(a, b) \in C$, and where the arbitrary constant $h_{0}$ has been put equal to one. With $K(x)=x^{-2}$ it follows (see Section 3 ) that the 
constants $h_{n}$ in the series (5.2) are given by

$$
h_{0}=1, \quad h_{1}=-\frac{1}{x} \text { and } h_{n}=0 \text { for } \mathrm{n} \geqslant 2
$$

Hence the function $\phi^{\prime}$ in (6.3) is given, from (5.2), by

$$
\phi^{\prime}(x, y)=\frac{1}{2 \pi} \mathscr{R}\left\{x \log \left(z-z_{0}\right)-\left(z-z_{0}\right) \log \left(z-z_{0}\right)+z-z_{0} \log \left(-z_{0}\right)\right\} .
$$

Elementary differentiation now yields the normal derivative in the form

$$
\frac{\partial \phi^{\prime}}{\partial n}=\left[\frac{1}{2 \pi} \mathscr{R}\left\{\frac{x}{z-z_{0}}\right\}\right] n_{1}+\left[\frac{1}{2 \pi} \mathscr{R}\left\{\frac{i x}{z-z_{0}}-i \log \left(z-z_{0}\right)\right\}\right] n_{2} .
$$

In the integral in (6.3) the only unknown is the normal derivative $\partial \phi / \partial n$. To obtain this derivative it is convenient first to let $\phi=1$ in (6.3) to obtain

$$
\lambda=+a \int_{C} x^{-2} \frac{\partial \phi^{\prime}}{\partial n} d s
$$

so that (6.3) may be written in the form

$$
\int_{C} x^{-2} \frac{\partial \phi}{\partial n} \phi^{\prime} d s=\int_{C} x^{-2}[\phi(x, y)-\phi(a, b)] \frac{\partial \phi^{\prime}}{\partial n} d s
$$

This equation may be employed to obtain numerical values of $\partial \phi / \partial n$ on various points on $C$ (see Clements and King [2] for details of the numerical procedure). Once this has been done, equations (6.3) with $\lambda=1$ yields $\phi$ at all interior points.

Values of $\partial \phi / \partial n$ calculated from (6.7) are given in Table 1 for the case when each side of the square is divided into two segments. It is apparent that for this simple

TABLE 1

Comparison of analytical and numerical solution for eight segments

\begin{tabular}{ccc}
\hline $\begin{array}{c}\text { Position } \\
(x, y)\end{array}$ & $\begin{array}{c}\text { Analytical } \\
\text { solution }\end{array}$ & $\begin{array}{c}\text { Numerical } \\
\text { solution }\end{array}$ \\
\hline$(1,1.75)$ & 0 & 0.006 \\
$(1,1.25)$ & 0 & -0.006 \\
$(1.25,1)$ & -1 & -1.001 \\
$(1.75,1)$ & -1 & -0.998 \\
$(2,1.25)$ & 0 & 0.001 \\
$(2,1.75)$ & 0 & -0.001 \\
$(1.75,2)$ & 1 & 0.998 \\
$(1.25,2)$ & 1 & 1.001 \\
\hline
\end{tabular}


problem the numerical results obtained from (6.7) compare favourably with those obtained from the analytical solution even though the number of boundary segments is small.

Although only the case $K(x)=x^{-2}$ has been considered in this example, it is clear that the case $K(x)=(\beta x+\delta)^{-2}$ may be treated in a very similar way with only minor modifications being needed in (6.4) and (6.5). Further, the case $K(x)=(\alpha x+\beta)^{2}$ would lead to even simpler forms for the auxiliary function $\phi^{\prime}$ and its normal derivative. Both of these forms for $K(x)$ contain two parameters which may be used to fit them to discrete numerical values of $K(x)$.

\section{Evaluation of $\phi^{\prime}$}

The example considered in the previous section does not provide any substantial information about how the auxiliary function $\phi^{\prime}$ should be evaluated numerically for more general forms for $K(x)$. We now consider this question and first examine the integral in (5.2). This may be written in the form

$$
\begin{gathered}
\int_{0}^{z}\left[\left(z-z_{0}\right)-\left(t-z_{0}\right)\right]^{n-1} \log \left(t-z_{0}\right) d t \\
=\sum_{r=0}^{n-1} \frac{(n-1) !(-1)^{r}}{r !(n-1-r) !}\left(z-z_{0}\right)^{n-1-r} \int_{0}^{z}\left(t-z_{0}\right)^{r} \log \left(t-z_{0}\right) d t \\
=\sum_{r=0}^{n-1} \frac{(n-1) !(-1)^{r}}{r !(n-1-r) !} \frac{\left(z-z_{0}\right)^{n-1-r}}{r+1}\left\{\left(z-z_{0}\right)^{r+1} \log \left(z-z_{0}\right)-\left(-z_{0}\right)^{r+1} \log \left(-z_{0}\right)\right. \\
\left.\quad-(r+1)^{-1}\left(z-z_{0}\right)^{r+1}+(r+1)^{-1}\left(-z_{0}\right)^{r+1}\right\} .
\end{gathered}
$$

Thus the integral in (5.2) may be replaced exactly by a finite sum which may be evaluated in a straightforward way for particular numerical values of $z, z_{0}$ and $n$.

Consider next the evaluation of $h_{n}(x)$. If $K(x)=(\alpha x+\beta)^{p}$, then the explicit forms given in (3.24) for $h_{n}(x)$ are relevant and the numerical evaluation of this function presents no special problem. When simple analytical expressions cannot be obtained for the $h_{n}(x)$, the derivatives in (3.7) may be replaced by appropriate finite difference formulas. Equation (3.7) then readily provides numerical values of $h_{n+1}(x)$ at a discrete set of points in the interval of interest.

Thus, if numerical values of $K(x)$ are given and no analytical form is known, then it is possible to proceed in two different ways. Firstly, an attempt may be made to fit one of the three parameter forms $(\alpha x+\beta)^{p}$ to the numerical data. If this can be done sufficiently accurately then the analytical forms (3.24) are available for the $h_{n}(x)$. 
Secondly, from (3.3) we have

$$
\Lambda(x)=\frac{1}{2 K} \frac{d^{2} K}{d x^{2}}-\frac{1}{4 K^{2}}\left(\frac{d K}{d x}\right)^{2}
$$

The derivatives on the right side may be replaced by finite difference formulas and hence numerical values of $\Lambda(x)$ obtained. Numerical values of $h_{n}(x)$ are then obtained from (3.7) by replacing the derivatives by finite difference formulas.

\section{References}

[1] S. Bergman, Integral operators in the theory of linear partial differential equations (Springer-Verlag, 1971).

[2] D. L. Clements and G. King, "A method for the numerical solution of problems governed by elliptic systems in the cut plane", J. Inst. Maths. Applics. 24 (1979), 81-93.

[3] D. L. Clements and C. Rogers, "Wave propagation in inhomogeneous elastic media with $(N+1)$ dimensional spherical symmetry", Canadian J. Phys. 52 (1974), 1246-1252.

[4] T. A. Cruse and J. C. Lachat (Eds.), Proceedings of the international symposium on innovative numerical analysis in applied engineering science (Versailles, France, 1977).

[5] T. A. Cruse and F. J. Rizzo (Eds.), Boundary integral equation method: Computational applications in applied mechanics (ASME Proceedings. AMD, Vol. II, 1975).

[6] O. C. Zienkiewicz, D. W. Kelly and P. Bettess, "The coupling of the finite element method and boundary solution procedures", Int. J. Num. Meth. Engng 11 (1977), 355-375.

Department of Applied Mathematics

University of Adelaide

Adelaide

South Australia 5000 